\title{
A NEW FLUORESCENCE-BASED METHOD TO MONITOR THE pH IN THE THYLAKOID LUMEN USING GFP VARIANTS
}

\author{
Hong Yang ${ }^{1}$, Xiaojun $\mathrm{Pu}^{1}, \mathrm{Lu} \mathrm{Wang}^{1}, \mathrm{Li} \mathrm{Liu}^{1}$ and Steven M. Theg ${ }^{2}$
}

\begin{abstract}
Summary
The $\Delta \mathrm{pH}$-dependent/Tat pathway is unique for using only proton motive force for driving proteins transport across the thylakoid membrane in chloroplasts among five different pathways. 9-aminoacridine fluorescence quenching is widely used to monitor the $\Delta \mathrm{pH}$ developed across the thylakoid membrane in the light. However, this method suffers from limited sensitivity to low $\Delta \mathrm{pH}$ values and to spurious fluorescence signals due to membrane binding. In order to develop a more sensitive method for monitoring the real $\mathrm{pH}$ of the thylakoid lumen without these problems we transformed Arabidopsis thaliana with a ratiometric $\mathrm{pH}$ sensitive GFP variant (termed pHluorin) targeted to the lumen by the prOE17 transit peptide. Positive transgenic plants displayed localization of pHluorin in the chloroplast by confocal microscopy, and fractionation experiments revealed that it is in the lumen. The pHluorin signal was the strongest in very young plants and diminished as the plants matured. The pHluorin released from the lumen displayed the expected fluorescence intensity changes in response to $\mathrm{pH}$ titration. The fluorescence signal in isolated chloroplasts responded to illumination in a manner consistent with light-dependent lumen acidification. Future experiments will exploit the use of this new pH-indicating probe of the thylakoid lumen to examine the influence of the thylakoid $\Delta \mathrm{pH}$ on ATP synthesis and protein transport.
\end{abstract}


1 Key words: Chloroplast, GFP, Genetically encoded biosensor, thylakoid lumen

$2 \mathrm{pH}$, proton dynamics

\section{1. Introduction}

5 Chloroplasts are most prominent organelles in green plants that are responsible for the reactions of

6 photosynthesis through which most of the planet's reduced carbon and atmospheric $\mathrm{O}_{2}$ are produced. In the light-dependent reactions, chloroplasts convert sunlight into chemical energy through the processes of electron transport and photophosphorylation. By the mid-1970s most researchers in bioenergetics accepted the basic tenets of the chemiosmotic coupling hypothesis, which states that the energy required for ATP synthesis was transiently stored in an

11 electrochemical proton gradient across the energy-transducing membrane [1, 2]. Despite its 12 stunning success, a number of labs question the classic chemiosmotic theory on whether the protonmotive force (pmf) arose from protons delocalized throughout the bulk aqueous volume or from protons located at the membrane surface [3].

As well studied, nucleus-encoded chloroplast proteins are synthesized in precursor form and import into the chloroplast via Toc and Tic complex [4]. Stromal chaperones and the stromal processing peptidase cleave the transit peptide from the incoming precursor protein driven by nucleotide triphosphate (NTP) hydrolysis. Then lumen located protein continues their step across the thylakoid membrane process via four different pathways: SRP (signal recognition particle) machinery, spontaneous, Sec and Tat pathways [4-6]. Precursors are recognized by the specific transport machinery through typical transit peptides. Not only native proteins but also foreign proteins fused by the transit peptide can be located to organelle on purpose. It was reported that targeting of EGFP within chloroplasts by cpTat pathway was successful [7].

Since 1966 originated from Aequorea Victoria, the wild type GFP cDNA has been modified by codon usage and removal of cryptic intron, to get higher intensity, improved solubility, less temperature sensitivity and different spectral characteristics for plants [8]. Engineered GFP protein is now widespread for plants (Av-GFP) for cellular localization, hormone movement, protein interaction, and so on [9-11]. Fluorescence intensity is also a useful indicator for comparing gene 
1 expression and activity of promoters or enhancers, cellular or extracellular ion dynamics [12].

2 Especially after pHluorins were engineered [13], it becomes more popular to measure proton activity and cytoplasmic free $\mathrm{Ca}$ concentration in root, pollen or cytoplasm under stress $[8,14,15]$. Ratiometric pHluorin is a pH-sensitive variants of Av-GFP, containing eight amino acid mutations (E132D, S147E, N149L, N164I, K166Q, I167V, R168H and L220F). The protein has double excitation wavelength at $395 \mathrm{~nm}$ and $475 \mathrm{~nm}$, displaying a reversible excitation ratio shift between pH 7.5 and 5.5 [13], So we think about locate this ratiometric pHluorin to the thylakoid lumen.

Previous measurements relating the $\Delta \mathrm{pH}$ component of the pmf to protein transport on the cpTat pathway were made using 9-aminoacridine (9-AA) fluorescence as readout of the $\Delta \mathrm{pH}$. However this assay is insensitive to values of the $\mathrm{pH}$ gradient lower than approximately $1.8 \mathrm{pH}$ units and to spurious fluorescence signals due to membrane binding [16]. In an effort to develop a more sensitive assay for the thylakoid transmembrane $\Delta \mathrm{pH}$, we constructed a novel $\mathrm{pH}$-sensitive GFP variant by overlapping the first 1-395 bp of sGFP (containing increasing solubility) with the last $324 \mathrm{bp}$ of ratiometric pHluorin containing all of the $\mathrm{pH}$ sensitive mutated amino acids together to get a new soluble and high quantum efficiency fluorescent protein. This modified ratiometric pHluorin was then driven by $35 \mathrm{~S}$ promoter and targeted to chloroplast lumen by fusing proe17 transit peptide before its cDNA. A series of experiments were performed to characterize the phenotype of the transformed A. thaliana and to demonstrate the proton gradient by lumen 19 localized pHluorin. Fluorescence images showed that pHluorin indeed locate at the thylakoid 20 luman. Luminal protein was released to prove that pHluorin was soluble in the lumen and 21 responded to different $\mathrm{pH}$. Confocal image was applied on mesophyll cells and guard cells of transformats during photosynthesis, to show the changes of fluorescence signal responding to

23 photosynthetic acidity. Consequently, it provides the possibilities to apply this novel approach 24 in the aspects of pmf in the chloroplast lumen. We also discussed the potential problems that may encounter when using this probe to do measurement. 
Ratiometric pHluorin gene was provided by the Memorial-Sloan Kettering Cancer Center [13].

2 This gene contains an aberrant splicing site in plants at the 5' part, which is the AGGTATTG sequence [17]. However, all pH-sensitive mutations exist in the downstream of the AGGTATTG sequence. The upstream before the AGGTATTG sequence was PCRed by primes proe17-sGFP-F/ sGFP-ph R394 from $s G F P$ gene [9], which was modified to be soluble in plants, was fused to fragment PCRed from Ratiometric pHluorin gene by primers sgfpPH394F/ NosEcor1R. This is named modified pHluorin gene. The transit peptide of proe17 from pea PCRed by primers Proe17Xma1F/ proe17-sGFP-R was fused to the 5' end of the modified pHluorin gene by overlap PCR. The cassette was inserted into the plasmid PBI121 after the CaMV35S promoter region. The resulting construct was transformed to wild-type A. thaliana ecotype Columbia (Col-0) plants using Agrobacterium tumefaciens EHA105 mediated method [18].

\subsection{Chloroplast fraction and protein extraction}

14 Intact chloroplasts were isolated from one week old, two weeks old, four weeks old plants growing on MS medium followed the protocol previous reported [19]. After osmotic shock for 5 min on ice in LS buffer (10mM MES, $\left.5 \mathrm{mM} \mathrm{MgCl}_{2}, \mathrm{pH} 6.5\right)$, the thylakoids were pelleted at 12000 $\mathrm{g}$ for $5 \mathrm{~min}$ at $4^{\circ} \mathrm{C}$. Thylakoids were then incubated in IB buffer (0.33M sorbitol, 50mM HEPS, pH8) with $0.1 \%$ Triton $\mathrm{X}-100$ for 3 minutes on ice in order to release lumenal proteins following the method previously reported (Hashimoto et al., 1996). After centrifuge at 144000g for $15 \mathrm{~min}$, the supernatant (lumenal protein) was precipitated by adding same amount of 30\%TCA

21 (Trichloroacetic acid) on ice for 15 minutes, followed by full speed centrifuge for 20 minutes. The pellet was resuspended in buffer (0.33M sorbitol, $16.7 \mathrm{mM}$ MES, $16.7 \mathrm{mM}$ tricine) PHed from 5.5

23 to 8.5 to the final concentration as $40 \mu \mathrm{g} / \mathrm{ml}$, seperately. Therefore, we had a series of lumenal 24 protein in different $\mathrm{PH}$ in hand for measuring the fluorescence intensity.

\section{$25 \quad 2.3$ Quantification of GFP fluorescence}

26 Fluorescence measurements were performed on a Fluorolog-3 spectrofluorometer (Jobin Yvon 27 Inc., Edison, NJ). For Figure 3, the intact chloroplasts isolated from transgenic plants were 28 fractioned to get luminal proteins as we described above in order to get the fluorescence intensity 
1 corresponding to $\mathrm{PH}$ as control. Emission wavelength for the excitation spectra was at $530 \mathrm{~nm}$.

2 For Figure 4, the intact chloroplasts isolated from transgenic plants were in IB and set to the final concentration as $40 \mu \mathrm{g}$ chlorophyll/ml. The sample was excited by $395 \mathrm{~nm}$ and $475 \mathrm{~nm}$ separately for a period of 90 seconds. From 10 to 60 seconds the acidic light was on for activating photosynthesis resulting in proton gradient.

\subsection{Cell Imaging}

8 For Figure 1, the isolated thylakoid images were observed using a Zeiss (PG-HITEC, Mem

9 Martins, Portugal) Axioskop 2 plus microscope equipped with a EBQ100 isolated fluorescent 10 lamp. For Figure 2, the in vitro chloroplast images were taken on a confocal laser scanning 11 microscope system (FV-1000; Olympus, Hamburg, Germany).

\section{Results} \\ 3.1 Expression pattern of ratiometric pHluorin in the transgenic Arabidopsis}

We transformed A. thaliana with a modified $\mathrm{pH}$-sensitive GFP variant (ratiometric pHluorin) targeted to the thylakoid lumen with the PrOE17 transit peptide. First we convinced the location of the ratiometric pHluorin by microscope images. As shown in Figure 1, the targeted pHluorin is indeed present in the thylakoid by microscope images of isolated thylakoids. Interestingly, we found that the fluorescence signal from chloroplast-targeted pHluorins are the strongest in younger leaves, and tend to be diminished in older leaves (Figure 1). In one week old plants, all of cells showed strong fluorescence signal in the thylakoids (Figure 1C). In two weeks old plants, most of the thylakoids still showed strong fluorescence signal (Figure 1G). In four weeks old plants, only small portion of the thylakoids showed strong fluorescence signal (Figure 1J). Only 6.3\%

25 1J). This result indicated that change of ratiometric pHluorin signal in thylakoid is associated with the leaf development. 
In order to know the distribution of ratiometric pHluorin in the transgenic Arabidopsis, the GFP

2 signal was investigated using intact leaves of one week old by confocal microscope (Figure 2).

3 RB-GFP was made by fusing Rubisco transit peptide with GFP, aiming to target GFP signal to

4 chloroplast stroma. Transgenic plants containing RB-GFP were used as control in chloroplasts

5 (Figure 2G). It clearly displayed that the chloroplasts in the guard cells had strong green

6 fluorescence (Figure 2A). However, the signal of the chloroplasts in the mesophyll cells was

7 inhomogeneous and only part of chloroplasts had green fluorescence. This small portion of

8 chloroplasts had strong green fluorescence but week chlorophyll autofluorescence (Figure 1D to

9 F). Since chlorophyll autofluorescence indicated the density of chlorophyll and thylakoid 10 development, this result suggests the expression of ratiometric pHluorin was associated with the 11 density of chlorophyll and thylakoid development. Therefore our next question is whether 12 ratiometric pHluorin in transgenic plants has original ratiometric properties.

\section{$14 \quad 3.3$ In vivo properties of pHluorin}

15 In order to explore in vivo properties of ratiometric pHluorin in transgenic plants, we liberated 16 ratiometric pHluorin from the lumen of one week old A. thaliana thylakoids, and demonstrate that this protein was indeed free in the soluble phase of the lumen and retains its $\mathrm{pH}$-sensitive

18 properties as shown in Figure 3A. The released proteins were titrated in different $\mathrm{pH}$ buffer from 195.5 to 8.5 and the fluorescence intensity was measured by fluoremeter. Excitation spectra of ratiometric pHluorin were taken at emission wavelength $530 \mathrm{~nm}$ for each pHed luminal protein.

21 The fluorescence intensity changed in a way of $\mathrm{pH}$-dependent. The fluorescence intensity in the left excitation peak at $395 \mathrm{~nm}$ decreased, while in the right peak at $475 \mathrm{~nm}$ increased during the $\mathrm{pH}$ decreased. A calibration curve based on the ratio of fluorescence intensities from pHluorin excited at 395 and $475 \mathrm{~nm}$ was shown in Figure 3B. It can be seen that this probe was indeed responsive in the range required to examine the $\mathrm{pH}$ changes in lumen.

The chloroplasts from transgenic A. thaliana with lumen-targeted ratiometric pHluorin were isolated and used to perform in vivo fluoremeter assays. From 10 to 60 seconds the red light was on for activating photosynthesis resulting in proton gradient. As Figure 4 showed, light-induced 
1 changes were shown when excitated in the peaks $395 \mathrm{~nm}$ and $475 \mathrm{~nm}$, and both curves were

2 deflected in the direction of acidification of the lumen. It is clear from these experiments that the

3 stable transgenic plants with a pHluorin targeted to the lumen was successful to report bulk $\mathrm{pH}$

4 changes in response to actinic illumination.

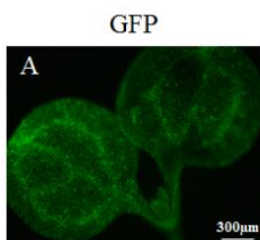

Chlorophyll

GFP Chlorophyll

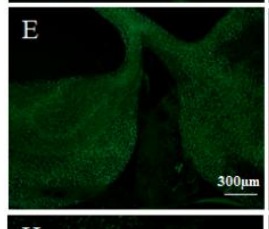

$\mathrm{F}$
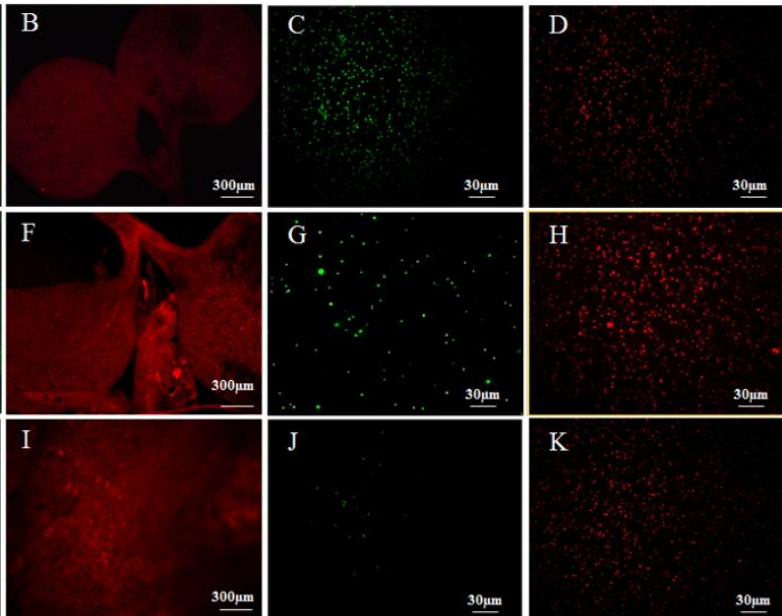

$\mathrm{H}$

$\mathrm{H}$
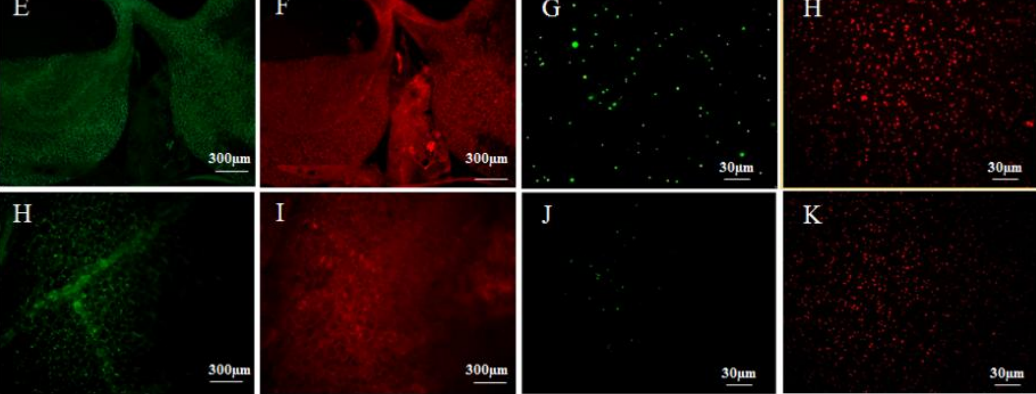

Figure 1. Ratiometric pHluorin signal change was associated with the leaf development. A to D, one week old seedling; E to H, two weeks old seedling; $\mathrm{H}$ to $\mathrm{K}$, four weeks old seedling. A, B, E, F, H, I, Fluorescence microscope images for the leaves at different stage. C, D,G, H, J, K, Fluorescence microscope images for the isolated thylakoids at different stage. The images were taken by a Zeiss (PG-HITEC, Mem Martins, Portugal) Axioskop 2 plus microscope equipped with a EBQ100 isolated fluorescent lamp.

11

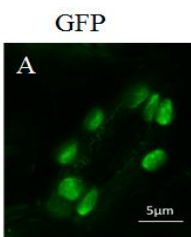

Chlorophyll Merged
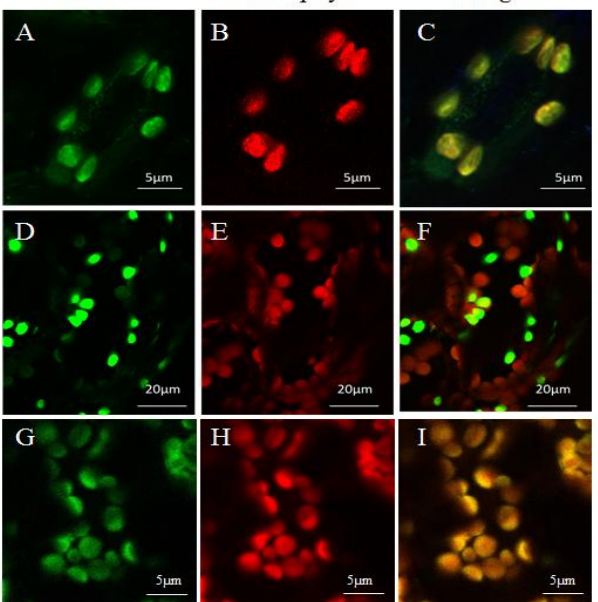

12 
1 Figure 2. Distribution of ratiometric pHluorin was inhomogeneous in transgenic A. thaliana leaves. A to C,

2 ratiometric pHluorin in the guard cells. D to F, ratiometric pHluorin in the mesophyll cells. G to I, RB-GFP in the

3 mesophyll cells as control. The images were taken on a confocal laser scanning microscope system (FV-1000;

4 Olympus, Hamburg, Germany ).

5

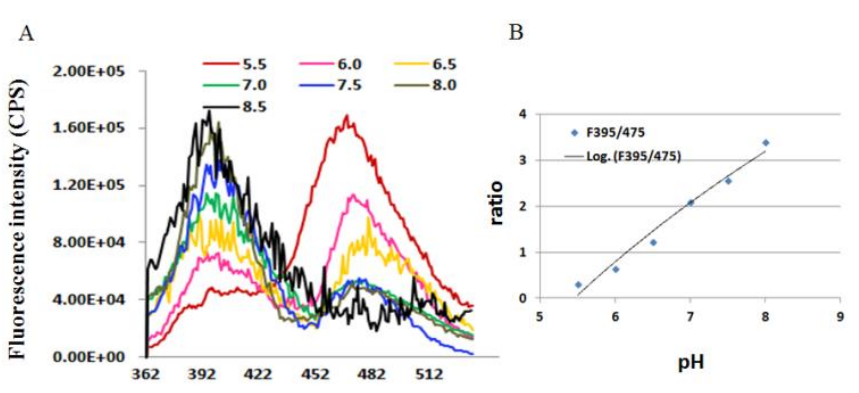

wavelength $(\mathrm{nm})$

8 Figure 3. Ratiometric pHluorin proteins released from thylakoid is $\mathrm{PH}$ dependent. Excitation spectra of ratiometric

9 pHluorin at emission wavelength 530nm, was taken at different pHs range from 5.5 to 8.5. Samples were proteins 10 released from the thylakoid in transgenic Arabidopsis at two weeks old. Fluorescence measurements were 11 performed on a Fluorolog-3 spectrofluorometer (Jobin Yvon Inc., Edison, NJ).

12

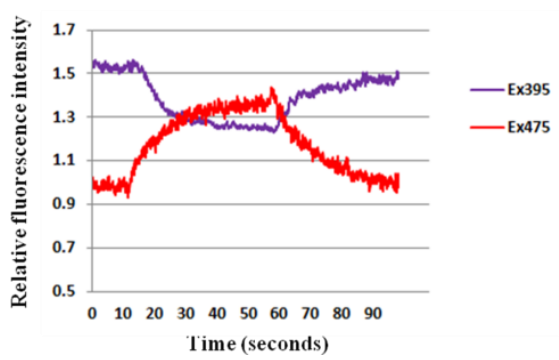

Figure 4. The fluorescence intensity of ratiometric pHluorin in isolated chloroplasts responded to illumination in a manner consistent with light-dependent lumen acidification. The samples were in import buffer at PH8.0, and excited by $395 \mathrm{~nm}$ and $475 \mathrm{~nm}$ separately. From 10 to 60 seconds the red light was on for activating photosynthesis resulting in proton gradient.

\section{Discussion}

In the present work we have examined the thylakoid translocation of a modified $\mathrm{pH}$-sensitive GFP named ratiometric pHluorin, which has double excitation wavelength at $395 \mathrm{~nm}$ and $475 \mathrm{~nm}$, 
1 displaying a reversible excitation ratio shift between $\mathrm{pH} 8.5$ and 5.5. The ratiometric pHluorin was

2 fused to transit peptides derived from a nucleus encoded chloroplast precursor protein proe17. Our

3 analyses showed that the ratiometric pHluorin is transported into the thylakoid lumen. However,

4 efficient thylakoid transport can only be achieved in the guard cells, which means all of the

5 chloroplasts involved in the guard cells showed fluorescence signal (Figure 2A). While In the

6 mesophyll cells, only a small portion of chloroplasts gave fluorescence signal. This small portion

7 of chloroplasts had strong green fluorescence but week chlorophyll autofluorescence (Figure 2),

8 which drove us think mature chloroplasts had no fluorescent proteins.

9 What is the reason for this phenomenon? Guard cells have much less chloroplasts compared 10 to mesophyll cells [20]. In most species studied, guard cells contain chloroplasts, which vary in 11 number depending upon the species [21]. Most species typically contain 10-15 chloroplasts per 12 guard cell, compared with 30-70 in a palisade mesophyll cell. Guard cell chloroplasts are often 13 smaller, with less thylakoid membrane stacks, and some are less well developed than those in 14 mesophyll cells, although these features vary across plant families. Considering the biological 15 properties of the guard cells and the mature chloroplasts, it appears reasonable to assume that the 16 ratiometric pHluorin proteins were digested in the lumen or did not fold in the right formation in 17 the mature chloroplasts.

18 Further experiments of calibration for ratiometric pHluorin proteins released from one week 19 old transgenic plants showed this protein was free in the soluble phase of the lumen and retained 20 its pH-sensitive properties (Figure 3). Also, fluorometer assay of isolated chloroplasts from 21 transgenic plants could report bulk pH changes in response to actinic illumination (Figure 4). We 22 assumed that the chloroplasts giving green fluorescence signal carried out photosynthesis 23 normally, and could be useful to the localization proton study for investigating the fundamental 24 questions about whether the protonmotive force (pmf) arose from protons delocalized throughout 25 the bulk aqueous volume or from protons localized at the membrane surface.

\section{Acknowledgments}


The constructs were created in our lab by modifying pHluorins, provided by the Memorial-Sloan Kettering Cancer Center, for use in plants. This work was supported by the Division of Chemical Sciences, Geosciences, and Biosciences, Office of Basic Energy Sciences of the U.S. Department of Energy through Grant DE-FG02-03ER15405 and 100 Talents Program of The Chinese Academy of Sciences and by National Natural Science Foundation of China (31571262).

\section{References}

[1] P. Mitchell, Chemiosmotic coupling in oxidative and photosynthetic phosphorylation. 1966, Biochimica et Biophysica Acta, 1807 (2011) 1507-1538.

[2] P. Mitchell, Chemiosmotic coupling in oxidative and photosynthetic phosphorylation, Biol Rev Camb Philos Soc, 41 (1966) 445-502.

[3] R.A. Dilley, On why thylakoids energize ATP formation using either delocalized or localized proton gradients - a Ca2+ mediated role in thylakoid stress responses, Photosynthesis Research, 80 (2004) 245-263.

[4] H.M. Li, C.C. Chiu, Protein transport into chloroplasts, Annual Review of Plant Biology, 61 (2010) 157-180.

[5] P. Jarvis, C. Robinson, Mechanisms of protein import and routing in chloroplasts, Current Biology, 14 (2004) R1064-1077.

[6] P. Jarvis, Targeting of nucleus-encoded proteins to chloroplasts in plants, New Phytologist, 179 (2008) 257-285.

[7] J.P. Marques, M.H. Schattat, G. Hause, I. Dudeck, R.B. Klösgen, In vivo transport of folded EGFP by the $\Delta$ pH/TAT-dependent pathway in chloroplasts of Arabidopsis thaliana, Journal of experimental botany, 55 (2004) 1697-1706.

[8] N. Moseyko, L.J. Feldman, Expression of pH-sensitive green fluorescent protein in Arabidopsis thaliana, Plant Cell Environ, 24 (2001) 557-563.

[9] W.L. Chiu, Y. Niwa, W. Zeng, T. Hirano, H. Kobayashi, J. Sheen, Engineered GFP as a vital reporter in plants, Current Biology, 6 (1996) 325-330.

[10] R. Waadt, P.K. Hsu, J.I. Schroeder, Abscisic acid and other plant hormones: Methods to visualize distribution and signaling, Bioessays, 37 (2015) 1338-1349.

[11] S. Okumoto, A. Jones, W.B. Frommer, Quantitative imaging with fluorescent biosensors, Annual Review of Plant Biology, 63 (2012) 663-706.

[12] D.M. Chudakov, S. Lukyanov, K.A. Lukyanov, Fluorescent proteins as a toolkit for in vivo imaging, Trends in Biotechnology, 23 (2005) 605-613.

[13] G. Miesenbock, D.A. De Angelis, J.E. Rothman, Visualizing secretion and synaptic transmission with pH-sensitive green fluorescent proteins, Nature, 394 (1998) 192-195.

[14] D. Gao, M.R. Knight, A.J. Trewavas, B. Sattelmacher, C. Plieth, Self-reporting Arabidopsis expressing $\mathrm{pH}$ and $[\mathrm{Ca} 2+]$ indicators unveil ion dynamics in the cytoplasm and in the apoplast 
under abiotic stress, Plant Physiology, 134 (2004) 898-908.

[15] S.J. Swanson, W.G. Choi, A. Chanoca, S. Gilroy, In vivo imaging of $\mathrm{Ca} 2+, \mathrm{pH}$, and reactive oxygen species using fluorescent probes in plants, Annual Review of Plant Biology, 62 (2011) 273-297.

[16] S.M. Theg, C. Tom, Measurement of the $\Delta \mathrm{pH}$ and electric field developed across Arabidopsis thylakoids in the light, Chloroplast Research in Arabidopsis: Methods and Protocols, Volume II, (2011) 327-341.

[17] J. Haseloff, K.R. Siemering, D.C. Prasher, S. Hodge, Removal of a cryptic intron and subcellular localization of green fluorescent protein are required to mark transgenic Arabidopsis plants brightly, Proceedings of the National Academy of Sciences, 94 (1997) 2122-2127.

[18] X. Zhang, R. Henriques, S.S. Lin, Q.W. Niu, N.H. Chua, Agrobacterium-mediated transformation of Arabidopsis thaliana using the floral dip method, Nature Protocols, 1 (2006) 641-646.

[19] P.H. Su, H.M. Li, Stromal Hsp70 Is Important for Protein Translocation into Pea and Arabidopsis Chloroplasts, Plant Cell, 22 (2010) 1516-1531.

[20] T. Lawson, Guard cell photosynthesis and stomatal function, New Phytologist, 181 (2009) 13-34.

[21] T. Lawson, K. Oxborough, J.I. Morison, N.R. Baker, The responses of guard and mesophyll cell photosynthesis to $\mathrm{CO} 2, \mathrm{O} 2$, light, and water stress in a range of species are similar, Journal of experimental botany, 54 (2003) 1743-1752. 